\title{
Identification of inhibitors regulating cell proliferation and FUS- DDIT3 expression in myxoid liposarcoma using combined DNA, mRNA, and protein analyses
}

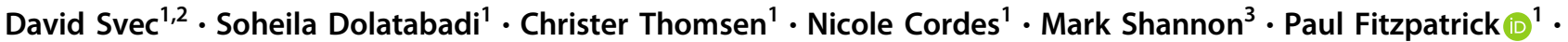 \\ Göran Landberg ${ }^{1} \cdot$ Pierre Åman $^{1} \cdot$ Anders Ståhlberg $\mathbb{D}^{1,4,5}$
}

Received: 29 August 2017 / Revised: 13 February 2018 / Accepted: 20 February 2018 / Published online: 27 March 2018

(c) The Author(s) 2018. This article is published with open access

\begin{abstract}
FUS-DDIT3 belongs to the FET (FUS, EWSR1, and TAF15) family of fusion oncogenes, which collectively are considered to be key players in tumor development. Even though over $90 \%$ of all myxoid liposarcomas (MLS) have a FUS-DDIT3 gene fusion, there is limited understanding of the signaling pathways that regulate its expression. In order to study cell proliferation and FUS-DDIT3 regulation at mRNA and protein levels, we first developed a direct cell lysis approach that allows DNA, mRNA, and protein to be analyzed in the same sample using quantitative PCR, reverse transcription quantitative GPCR and proximity ligation assay, respectively. We screened 70 well-characterized kinase inhibitors and determined their effects on cell proliferation and expression of FUS-DDIT3 and FUS at both mRNA and protein levels in the MLS 402-91 cell line, where twelve selected inhibitors were evaluated further in two additional MLS cell lines. Both FUSDDIT3 and FUS mRNA expression correlated with cell proliferation and both transcripts were co-regulated in most conditions, indicating that the common 5' FUS promotor is important in transcriptional regulation. In contrast, FUS-DDIT3 and FUS protein levels displayed more cell line dependent expression. Furthermore, most JAK inhibitors caused FUSDDIT3 downregulation at both mRNA and protein levels. In conclusion, defining factors that regulate FUS-DDIT3 expression opens new means to understand MLS development at the molecular level.
\end{abstract}

These authors contributed equally: David Svec, Soheila Dolatabadi.

Electronic supplementary material The online version of this article (https://doi.org/10.1038/s41374-018-0046-3) contains supplementary material, which is available to authorized users.

Anders Ståhlberg

anders.stahlberg@gu.se

1 Sahlgrenska Cancer Center, Department of Pathology and Genetics, Institute of Biomedicine, Sahlgrenska Academy at University of Gothenburg, Medicinaregatan 1F, 41390 Gothenburg, Sweden

2 Laboratory of gene expression, Institute of Biotechnology AS CR, BIOCEV, Prumyslova 595, 25250 Vestec by Prague, Czech Republic

3 Genetic Sciences Division, Thermo Fisher Scientific, 180 Oyster Point Blvd., South San Francisco, CA 94080, USA

4 Wallenberg Centre for Molecular and Translational Medicine, University of Gothenburg, Gothenburg, Sweden

5 Department of Clinical Pathology and Genetics, Sahlgrenska University Hospital, 41345 Gothenburg, Sweden

\section{Introduction}

Measuring cell proliferation, mRNA expression and protein levels in a sample typically requires three different methodologies that have their own inherent limitations. Most methodologies for quantifying mRNA levels require extraction steps that are associated with material losses and variable extraction efficiencies. Several techniques for measuring proteins, including western blots, require high cell numbers to generate reliable data. Hence, analyzing cell proliferation, mRNA expression, and protein levels in high throughput is labor intensive and often impractical. However, recent advances in molecular techniques open up new possibilities to quantify several types of analytes, including DNA, RNA, and protein, in the same sample, i.e., pluriomics [1]. Furthermore, through the use of direct lysis without further sample processing, higher analyte yields are generated, thereby, allowing smaller sample sizes to be used, even single-cells [2]. Multianalyte analysis on small sample sizes is particularly useful when attempting to evaluate many conditions where protein and mRNA levels are expected to 


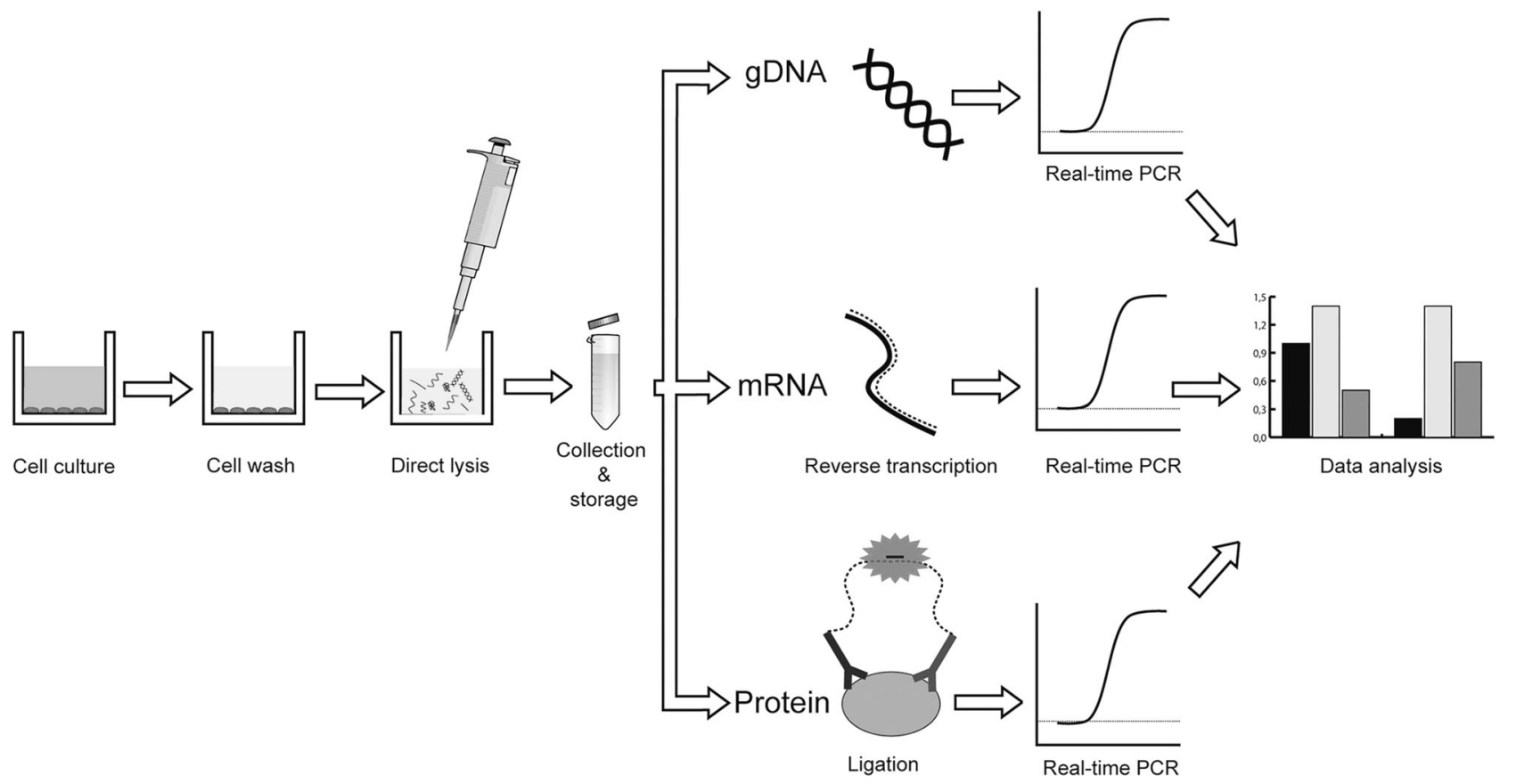

Fig. 1 Schematic overview of direct lysis followed by cell proliferation, mRNA, and protein analysis

be differentially regulated, such as studying the effect of kinase inhibitors on fusion oncogene expression.

Fusion oncogenes are common in many cancer types and are often driver mutations in tumor development. The FET family of oncogenes (FUS, EWSR1, and TAF15) comprises more than twenty distinct oncogenes and defines a subgroup of sarcomas and leukemia, where myxoid liposarcoma (MLS) and Ewing sarcoma are the most common entities [3-5]. The FET fusion oncogenes always include a $5^{\prime}$ part of FUS, EWSR1, or TAF15 fused to one of many alternative genes that encode DNA-binding transcription factors [3-5]. The $3^{\prime}$ transcription factor partners are tumor type-specific, while the $5^{\prime}$ FET partners may replace each other. More than $90 \%$ of all MLS cases carry FUS-DDIT3, resulting from the $\mathrm{t}(12 ; 16)$ translocation. Although the FUS-DDIT3 gene fusion has been implicated in MLS development, its transforming mechanism is unknown $[4,6,7]$.

The normal FUS protein is abundantly expressed in most tissues [8], while DDIT3 expression is highly regulated and only expressed in specific tissues and under stress conditions [9]. At the mRNA level, the 5' FUS promotor regulates FUS-DDIT3 transcription, while FUS-DDIT3 mRNA stability depends on the $3^{\prime}$ DDIT3 sequence [10]. At the protein level, FUS interactions rather than DDIT3 interactions determine the FUS-DDIT3 expression and stability. Furthermore, FUS-DDIT3 half-life is shorter and expression level lower than for normal FUS at both mRNA and protein [10]. To date, little is known about the factors and signaling pathways that act upstream of FUS-DDIT3 to regulate its expression.
Here, we developed an approach to quantify cell proliferation, mRNA expression, and protein levels in the same sample using direct lysis (Fig. 1). To assess cell proliferation we quantified the amount of genomic DNA (gDNA) using quantitative real-time PCR (qPCR). For mRNA and protein analyses, we applied reverse transcription (RT) qPCR and proximity ligation assay (PLA), respectively. The final readout for all analyses was cycle of quantification (Cq) values, which simplified and streamlined data analysis. We applied this multi-analyte approach to screen a kinase inhibitor library to identify signaling pathways that affect cell proliferation and regulate the expression of FUS-DDIT3 and FUS at mRNA and protein levels in the MLS 402-91 cell line. Finally, we tested twelve selected inhibitors in two additional MLS cell lines in order to assess their responses in a more generalized MLS context.

\section{Materials and methods}

\section{Cell culture and drug screening}

All cell lines, MLS 402-91, MLS 2645-94, MLS 1765-92, and HT1080, were cultured in RPMI 1640 GlutaMAX medium supplemented with $5 \%$ fetal bovine serum and 100 $\mathrm{U} / \mathrm{mL}$ penicillin and $100 \mu \mathrm{g} / \mathrm{mL}$ streptomycin (all Thermo Fisher Scientific). Cell passaging was performed using $0.25 \%$ trypsin and $0.5 \mathrm{mM}$ EDTA (Invitrogen).

To evaluate PLA specificity we used HT1080 cells that transiently expressed FUS-DDIT3-EGFP or FUS-EGFP as 

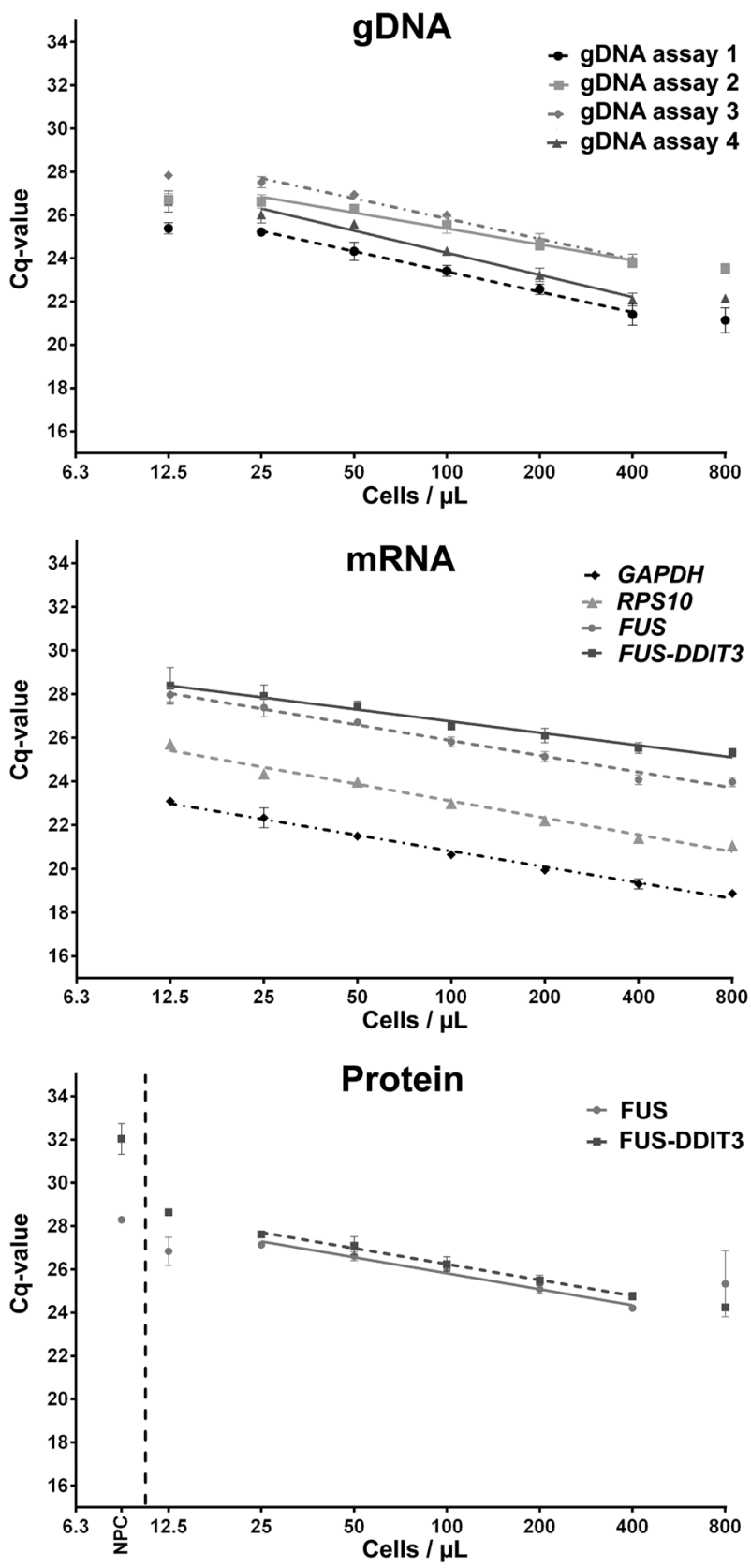

Fig. 2 Dynamic range of multi-analyte analysis using direct lysis. 2500-160,000 MLS 402-91 cells were lysed in $200 \mu \mathrm{L}$ BSA direct lysis buffer. The dynamic range of four gDNA assays, four mRNA assays and two PLAs were assessed. The linear fit is shown for respective assays. The average linear slope is: gDNA assays $=-3,01$; mRNAs $=-2,29$; proteins $=-2,44$. The performance of each assay is shown in Supplementary Table 2. The numbers of cell concentrations included in the linear regression were based on visual inspection and residual plots. The negative protein control (NPC) shows the background ligation for each PLA. Data are shown as Cq mean $\pm \mathrm{SD}, n=3$

described [11]. Briefly, transfections were performed with FuGENE 6 Transfection Reagents, according to the manufacturer's instruction (Roche). After $24 \mathrm{~h}$, cells were harvest in Protein Quant Sample Lysis Kit buffer (Applied Biosystems).
To identify signaling pathways that regulate cell proliferation and FUS-DDIT3 regulation, a library of kinase inhibitors targeting stem cell signaling was applied (\#L2100, Selleck chemical). Seventeen thousand MLS 402-91 cells were seeded per well using 96-well plates (Sigma-Aldrich). After $24 \mathrm{~h}, 10 \mu \mathrm{M}$ of each inhibitor was added to four wells in different 96-well plates. After $24 \mathrm{~h}$, inhibitor-treated cells were harvested using direct lysis. For the follow-up experiments, MLS 402-91, 2645-94, and 1765-92 cells were treated with $2.5 \mu \mathrm{M}$ inhibitor in the same manner as the screening experiment descried above. Each inhibitor was added to three independent wells in the same plate for the respective cell lines and the experiments were repeated 3-4 times using independent cell passages.

\section{Direct cell lysis}

Cells were washed once with $100 \mu \mathrm{L}$ PBS and then directly lysed in $200 \mu \mathrm{L}$ lysis buffer containing $1 \mu \mathrm{g} / \mu \mathrm{L}$ bovine serum albumin supplied in $2.5 \%$ glycerol (BSA direct lysis buffer, \#B14 diluted 1:20 in water, Thermo Fisher Scientific). Lysates were stored in $-80{ }^{\circ} \mathrm{C}$ and mixed by pipetting prior use. As quality controls, the same number of DNA spike (I or III) and RNA spike II molecules (all TATAA Biocenter) were added to each sample.

To test the effect of direct lysis volume, 10,000 cells were lysed in 50,100,200, $400 \mu \mathrm{L}$ using either BSA direct lysis buffer or PLA lysis buffer. PLA lysis buffer was prepared using the Protein Quant Sample Lysis Kit (Applied Biosystems), according to the manufacturer's instructions.

To evaluate cell proliferation using gDNA and direct lysis we performed the alamarBlue Cell Viability assay according to the manufacturers' instructions (Thermo Fischer Scientific). 2500-16,000 cells were either seeded into 96-well plates (Sigma-Aldrich) or lysed in $200 \mu \mathrm{L}$ BSA direct lysis buffer. The alamarBlue fluorescence signal was analyzed about $4 \mathrm{~h}$ after seeding using a VICTOR3 microplate reader (PerkinElmer). Wells with culture medium and alamarBlue only were used as controls. The time interval between cell seeding and cell proliferation analysis enabled cells to attach on the plate surface.

In order to validate the dynamic range of direct lysis for DNA, mRNA and protein analysis, we performed a twofold serial dilution of the cell lysate, starting with 160,000 MLS 402-91 cells in $200 \mu \mathrm{L}$ down to up to 2500 cells (Fig. 2).

\section{Proximity ligation assay}

To generate sensitive and specific PLA probes against FUS-DDIT3 and FUS, several antibodies targeting different epitopes were evaluated (Supplementary Figure 1). 
Antibody biotinylation was performed with either the EZLink Sulfo-NHS-LC-Biotin (Thermo Scientific) or the APEX Biotin-XX Antibody Labeling Kit (Invitrogen) followed by dialysis using Slide-A-Lyzer MINI Dialysis Unit (Thermo Scientific) according to the TaqMan Protein Assays Probe Development Protocol (Applied Biosystems). Biotinylated antibodies were used to generate PLA probes using the TaqMan Protein Assays Oligo Probe Kit, according to the manufacturer's instructions (Applied Biosystems). All candidate PLA probes passed the forced proximity ligation test. To test PLA probe sensitivity and specificity we quantified FUS-DDIT3 and FUS expression in HT1080 cells and compared their expression level to HT1080 cells expressing FUS-DDIT3-EGFP and FUSEGFP, respectively (Supplementary Figure 1). These initial PLA experiments were performed with TaqMan Protein Assay reagents as described [1]. For all other PLA experiments, i.e., inhibitor screening and validation experiments, the Assay Probe Dilution Buffer II and the TaqMan Protein Assay II (both Applied Biosystems) were used. Briefly, 2 $\mu \mathrm{L}$ of $80 \mathrm{pmol} / \mathrm{L}$ PLA probe solution was mixed with $2 \mu \mathrm{L}$ of lysed cells and incubated at $4{ }^{\circ} \mathrm{C}$ overnight. For the FUS PLA assay, cell lysates were 1-10 times additionally diluted using direct lysis buffer to keep the amount of FUS in the dynamic range of the assay. Working on ice, $16 \mu \mathrm{L}$ of freshly prepared ligation qPCR mix was added, containing: 1× Protein Assays Fast Master Mix, 1× Universal PCR Assay II, $1 \times$ DNA Ligase II, and water to a final volume of $20 \mu \mathrm{L}$ (all Applied Biosystems). Thermocycling was performed with a CFX384 Touch real-time cycler (Bio-Rad). The temperature profile for ligation was $25^{\circ} \mathrm{C}$ for $5 \mathrm{~min}$. Quantitative PCR followed immediately: $95^{\circ} \mathrm{C}$ for $20 \mathrm{~s}, 40$ cycles of amplification $\left(94^{\circ} \mathrm{C}\right.$ for $1 \mathrm{~s}$ and $60^{\circ} \mathrm{C}$ for $20 \mathrm{~s}$ ). Background ligation was measured using no protein controls with direct lysis buffer containing no cell lysate.

The ab70381 antibody selected for final FUS PLA probes was evaluated by western blot analysis. Briefly, MLS cells were collected in RIPA lysis buffer supplemented with $1 \times$ Halt Protease and Phosphatase Inhibitor Cocktail (both Thermo Fisher Scientific). SDS-PAGE was performed with the NuPAGE system (Thermo Fisher Scientific), according to the manufacturer's instructions. Membranes were blocked with either 5\% skim milk before adding the antibody against FUS (Merck Chemicals) or 5\% bovine serum albumin before adding the antibody against GAPDH (Sigma-Aldrich) in TBS-T buffer $(50 \mathrm{mM}$ Tris$\mathrm{HCl}, \mathrm{pH} 6.8,50 \mathrm{mM} \mathrm{NaCl}, 0.05 \%$ Tween 20; all SigmaAldrich). Membranes were incubated overnight with primary antibodies against; FUS C-terminal (\#ab70381, Abcam, diluted 1:10,000) and GAPDH (\#ab9484, Abcam, diluted 1:1000). Detection was performed with Horseradish Peroxidase conjugated secondary antibodies (32430 or 32460, Thermo Fisher Scientific, diluted 1:1000) combined with Supersignal West Dura Extended Duration Substrate (Thermo Fisher Scientific). Chemiluminescence was detected using ImageQuant LAS 4000 mini (GE Healthcare Life Sciences).

\section{Reverse transcription}

Reverse transcription was performed using GrandScript cDNA Synthesis kit (TATAA Biocenter) in $10 \mu \mathrm{L}$ reactions, including $2 \mu \mathrm{L} 5 \times$ GrandScript RT reaction mix, $0.5 \mu \mathrm{L}$ GrandScript RT enzyme, $5.5 \mu \mathrm{L}$ water and $2 \mu \mathrm{L}$ of lysate. RT was performed at $25^{\circ} \mathrm{C}$ for $5 \mathrm{~min}, 42^{\circ} \mathrm{C}$ for $30 \mathrm{~min}$ and terminated at $85^{\circ} \mathrm{C}$ for $5 \mathrm{~min}$. Samples were diluted with $50 \mu \mathrm{L}$ TE buffer, $\mathrm{pH} 8.0$ (Invitrogen) and stored $-20^{\circ} \mathrm{C}$ before downstream qPCR.

\section{Quantitative PCR}

For gDNA analysis each $10 \mu \mathrm{L}$ qPCR contained $1 \mu \mathrm{L}$ cell lysate. For mRNA analysis each $6 \mu \mathrm{L}$ qPCR contained $2 \mu \mathrm{L}$ diluted cDNA. All qPCRs were performed with CFX384 Touch real-time cycler (Bio-Rad) and each reaction contained 1× TATAA SYBR GrandMaster Mix (TATAA Biocenter) and $400 \mathrm{nM}$ of each primer. Assay information and primer sequences are shown in Supplementary Table 1. The temperature profile was $95^{\circ} \mathrm{C}$ for 1 min followed by 45 cycles of amplification $\left(95^{\circ} \mathrm{C}\right.$ for $3 \mathrm{~s}, 58^{\circ} \mathrm{C}$ for $30 \mathrm{~s}$, and 72 ${ }^{\circ} \mathrm{C}$ for $10 \mathrm{~s}$ ). The formation of expected PCR products was confirmed for each assay by agarose gel electrophoresis and all qPCRs were analyzed by melting curve analysis. PCR efficiency was determined with standard curve analysis. To identify reference assays, including both gDNA and mRNA assays, NormFinder was used as described [12, 13]. To determine cell proliferation and to normalize relative mRNA and protein expression we used different gDNA assays for respective MLS cell lines and experiments (Supplementary Table 2). Quantitative PCR and data analyses were performed with the $2^{-\Delta \Delta \mathrm{Cq}}$ method in agreement with the minimum information for publication of quantitative real-time pcr experiments (MIQE) guidelines [14].

\section{Results}

\section{Generation of FUS-DDIT3 and FUS specific PLAs}

To quantify FUS-DDIT3 and FUS proteins we generated several PLAs targeting different epitopes of each protein (Supplementary Figure 1A and B). To test sensitivity and specificity of each PLA we compared the PLA signal, i.e., Cq-value, of HT1080 cells with or without transiently expressed FUS-DDIT3-EGFP or FUS-EGFP to that of negative protein controls (NPCs) (Supplementary 
Figure 1C). Negative protein controls represent the background signal generated by unspecific ligation between the two PLA probes without any proteins. Normally cultured HT1080 cells endogenously express FUS, but neither FUSDDIT3 nor DDIT3. For FUS-DDIT3, the PLA using the 15204-1-AP antibody targeting DDIT3 displayed highest specificity and sensitivity, since a negligible background signal was detected in HT1080 cells compared to NPCs. In addition, the difference in signal between HT1080 cells expressing FUS-DDIT3 compared to wild-type HT1080 was also highest for the 15204-1-AP antibody based PLA probe pair, among the 16 PLA probe combinations tested. For FUS, the optimal PLA probe pair out of 6 candidates, was based on the ab70381 and the NBP1-50623 antibodies. The FUS PLA detects endogenous FUS protein but not the N-terminal part of FUS-DDIT3, except for the FUS-DDIT3 Type VI that is expressed in MLS 1765-92 cells that includes almost the entire FUS protein (Supplementary Figure 1A). Two of the selected antibodies (15204-1-AP and NBP1-50623) were used for western blots in an earlier study [10] whilst the western blot specificity of the third antibody (ab70381) is shown in Supplementary Figure 2.

\section{Development of a direct lysis approach to quantify DNA, RNA, and protein in the same sample}

We have previously shown that DNA, RNA, and protein can be quantified in single-cells using various direct lysis approaches [1, 2, 10, 15]. Based on our previous data, we used bovine serum albumin supplied in glycerol (BSA\#14, diluted 1:20 in water, Thermo Fisher Scientific) as direct lysis buffer (BSA direct lysis buffer). Compared to DNA and RNA, proteins are highly variable in terms of physical properties. Hence, lysis conditions may affect various proteins differently. Therefore, to test how efficiently we could quantify FUS-DDIT3 and FUS we also tested a standard PLA lysis buffer (Protein Quant Sample Lysis Buffer, Applied Biosystems). Supplementary Figure 3 shows that the BSA direct lysis buffer is superior the PLA lysis buffer to quantify DNA, while they were equally suitable for quantifying mRNA. Analyses of spiked in DNA and RNA control molecules showed that BSA direct lysis buffer caused neither nucleic acids degradation nor enzymatic inhibition. For protein analysis, the PLA lysis buffer outperformed BSA direct lysis buffer for detecting FUS, while the difference for FUS-DDIT3 was smaller. However, BSA direct lysis still allowed reliable FUS quantification. Based on these data we chose to use the BSA direct lysis buffer, since it allowed DNA, RNA and protein analysis from the same sample. To determine the dynamic range we generated a standard curve, containing 12.5-800 MLS 402-91 cells per $\mu \mathrm{L}$ BSA direct lysis buffer, quantifying gDNA, mRNA, and protein (Fig. 2). Genomic DNA, mRNA, and protein could be detected within the whole tested dynamic range, but the linear response for gDNA and protein analysis ranged between 25 and 400 cells per $\mu \mathrm{L}$ lysate. For mRNA the linear response ranged between 12.5 and 400 cells per $\mu \mathrm{L}$ lysate.

To evaluate the use of BSA direct lysis followed by gDNA quantification to assess cell proliferation we compared our assay to the alamarBlue cell viability assay. Supplementary Figure 4 shows the linear response between the amount of gDNA quantified by GPCR and the fluorescence signal generated by the alamarBlue cell viability assay for MLS 402-91, 2645-94, and 1765-92.

\section{Identification of kinase inhibitors affecting cell proliferation}

To identify signaling pathways that affect cell proliferation and regulate FUS-DDIT3 and FUS expression at mRNA and protein level we treated MLS 402-91 cells with 70 well-characterized kinase inhibitors at a concentration of 10 $\mu \mathrm{M}$ (Table 1). After $24 \mathrm{~h}$ treatment, cells were collected in BSA direct lysis buffer and the lysates were forwarded to gDNA, mRNA, and protein analyses as shown in Fig. 1.

To assess cell proliferation we determined the number of cells by analyzing three gDNA assays using qPCR targeting different chromosomes, averaging their Cq-values. We applied the NormFinder algorithm to determine which gDNA assays to use (Supplementary Table 2). Figure 3a shows the cell proliferation for all inhibitors in relation to control cells. Supplementary Figure 5 shows that DNA was reliably quantified in each condition using DNA spike molecules. Sixty-two inhibitors decreased cell proliferation, while 8 inhibitors increased cell proliferation. Twenty-two inhibitors displayed $>$ two-fold reduction in cell proliferation and inhibitors 1-7 caused over 20-fold decrease in cell proliferation and massive cell death. No inhibitor increased cell proliferation $>$ two-fold.

\section{Identification of kinase inhibitors regulating FUS- DDIT3 and FUS expression at mRNA and protein level}

Next, we quantified FUS-DDIT3 and FUS mRNA expression using RT-qPCR. Supplementary Figure 5 shows that RNA was reliable quantified in each condition using RNA spike molecules. Traditionally, gene expression data are normalized against stably expressed reference genes. Here, we instead normalized the FUS-DDIT3 and FUS mRNA expression against the number of cells, i.e., gDNA signal. We applied the NormFinder algorithm to test the suitability of using gDNA instead of reference mRNAs to normalize data (Supplementary Figure 6 and Supplementary Table 2). Hence, we normalized FUS-DDIT3 and FUS mRNA 
Table 1 Kinase inhibitors

\begin{tabular}{|c|c|c|c|c|c|}
\hline Identification & Inhibitor & Target & Identification & Inhibitor & Target \\
\hline 1 & Ro 31-8220 & pan-PKC & 36 & LGK-974 & PORCN \\
\hline 2 & $T G 101348$ & $J A K 2$ & 37 & AG-490 & EGFR \\
\hline 3 & BMS-833923 & Smoothened & 38 & XAV-939 & Wnt $/ \beta$-catenin \\
\hline 4 & $\begin{array}{l}\text { NVP-BSK805 } \\
2 \mathrm{HCl}\end{array}$ & JAK2 & 39 & CHIR-98014 & GSK- $3 \alpha / \beta$ \\
\hline 5 & WP1066 & JAK2/ STAT3 & 40 & Hesperetin & TGF- $\beta$ \\
\hline 6 & $T G 101209$ & $J A K 2$ & 41 & LY2784544 & JAK2 \\
\hline 7 & AT9283 & $J A K 2 / 3$ & 42 & 1-Azakenpaullone & GSK-3 $\beta$ \\
\hline 8 & CEP-33779 & JAK2 & 43 & RKI-1447 & ROCK $1 / 2$ \\
\hline 9 & Go 6983 & pan-PKC & 44 & MK-0752 & $\gamma$-secretase \\
\hline 10 & $K 02288$ & Type I BMPR & 45 & DAPT (GSI-IX) & $\gamma$-secretase \\
\hline 11 & $A Z 960$ & $J A K 2$ & 46 & Enzastaurin & PKC $\beta$ \\
\hline 12 & Fasudil & ROCK2 & 47 & XL019 & JAK2 \\
\hline 13 & CHIR-99021 & $G S K-3 \alpha / \beta$ & 48 & LY364947 & TGF $\beta$ R-I \\
\hline 14 & Quercetin & $\mathrm{PI} 3 \mathrm{~K}$ & 49 & LY2157299 & TGF $\beta R-I$ \\
\hline 15 & PF-5274857 & Smoothened & 50 & Vismodegib & Hedgehog \\
\hline 16 & YO-01027 & $\gamma$-secretase & 51 & IWR-1-endo & Wnt $/ \beta$-catenin \\
\hline 17 & Sotrastaurin & pan-PKC & 52 & Avagacestat & $\gamma$-secretase \\
\hline 18 & CYT387 & $J A K 1 / 2$ & 53 & Pirfenidone & TGF $\beta$ \\
\hline 19 & TWS119 & 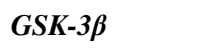 & 54 & LY411575 & $\gamma$-secretase \\
\hline 20 & IWP-L6 & PORCN & 55 & $\mathrm{Y}-276322 \mathrm{HCl}$ & ROCK1 \\
\hline 21 & ICG-001 & $\mathrm{Wnt} / \beta$-catenin & 56 & Purmorphamine & Smoothened \\
\hline 22 & Astragaloside A & TGF $\beta /$ Smad & 57 & S-Ruxolitinib & JAK1/2 \\
\hline 23 & SB415286 & GSK $3 \alpha / \beta$ & 58 & SB216763 & GSK- $3 \alpha / \beta$ \\
\hline 24 & KY02111 & $\mathrm{Wnt} / \beta$-catenin & 59 & SANT-1 & Smoothened \\
\hline 25 & Ruxolitinib & $\mathrm{JAK} 1 / 2$ & 60 & SB525334 & $\begin{array}{l}\text { TGF } \beta R-I \\
\text { (ALK5) }\end{array}$ \\
\hline 26 & SB505124 & $\begin{array}{l}\text { TGF } \beta \text { R (ALK4/ } \\
\text { 5) }\end{array}$ & 61 & Thiazovivin & ROCK \\
\hline 27 & Baricitinib & $J A K 1 / 2$ & 62 & Tofacitinib & JAK3 \\
\hline 28 & LY2109761 & TGF- $\beta$ R-I/II & 63 & Wnt-C59 (C59) & PORCN \\
\hline 29 & RepSox & TGFßR-1/ALK5 & 64 & AZD1080 & GSK- $3 \alpha / \beta$ \\
\hline 30 & AZD2858 & GSK-3 & 65 & SB431542 & ALK5 \\
\hline 31 & Tideglusib & GSK-3 $\beta$ & 66 & LDE225 & Smoothened \\
\hline 32 & LY2811376 & $\beta$-secretase & 67 & Tofacitinib & JAK3 \\
\hline 33 & Indirubin & GSK-3 $\beta$ & 68 & BIO & GSK- $3 \alpha / \beta$ \\
\hline 34 & GW788388 & ALK5 & 69 & WHI-P154 & JAK3 \\
\hline 35 & GSK429286A & ROCK1/2 & 70 & Semagacestat & $\gamma$-secretase \\
\hline
\end{tabular}

Inhibitors tested in all three MLS cell lines are shown in italic and bold font styles.

expression with the same gDNA assays as used to assess cell proliferation. Figure $3 \mathrm{~b}$ and Table 2 show that FUSDDIT3 and FUS were highly co-regulated. Furthermore, both FUS-DDIT3 and FUS levels correlated weakly but positively with cell proliferation. 21 and 18 inhibitors generated $\geq 2$-fold FUS-DDIT3 and FUS regulation, respectively. No reliable data for inhibitors 1-7 were obtained, due to cell death.
To quantify FUS-DDIT3 and FUS protein expression we used PLA. Protein expression was normalized against the same three gDNA assays that were used to determine cell proliferation and normalize relative mRNA expression. Figure $3 \mathrm{c}$ and Table 2 show that FUS-DDIT3 and FUS display variable expression patterns between each other for most inhibitors. FUS-DDIT3, but not FUS, showed a weak correlation with cell proliferation. The expression of 

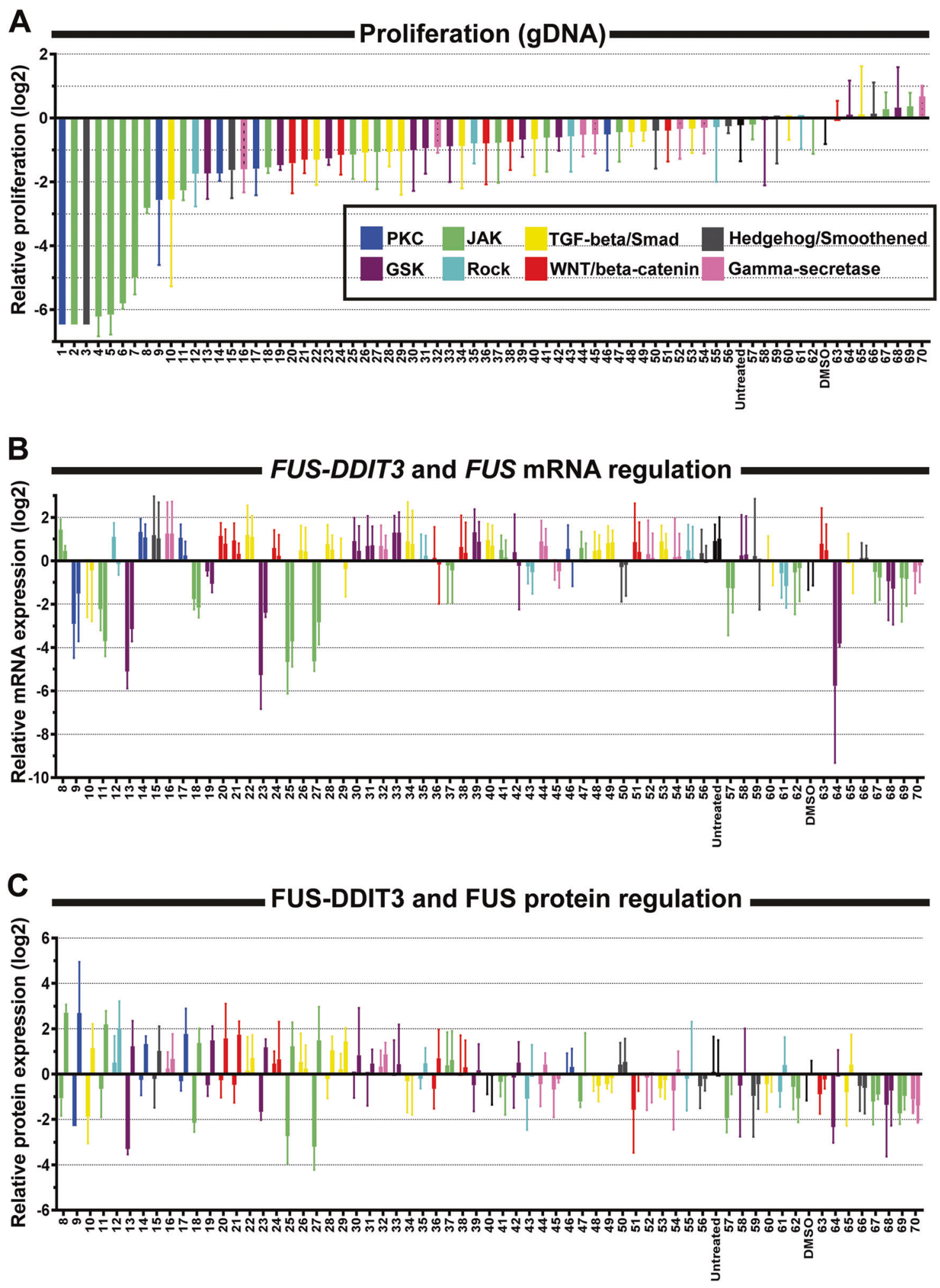

Fig. 3 Kinase inhibitor screening. MLS 402-91 cells were treated with 70 kinase inhibitors $(10 \mu \mathrm{M})$ and compared to treatment controls (DMSO) and untreated cells. Cells from four wells using four different 96-well plates were analyzed. A Cell proliferation. The DMSO control was arbitrarily set to a value of zero. Mean \pm SD is shown, $n=4$. B FUS-DDIT3 and FUS mRNA expressions. The DMSO controls were arbitrarily set to a value of zero. FUS-DDIT3 (left) and FUS (right) expressions are shown pairwise for each inhibitor. Data for inhibitors 1 to 7 were excluded due to massive cell death. Mean \pm SD is shown, $n$ $=4$. C FUS-DDIT3 and FUS protein expressions. The DMSO controls were arbitrarily set to a value of zero. FUS-DDIT3 (left) and FUS (right) expressions are shown pairwise for each inhibitor. Data for inhibitors 1 to 7 were excluded due to massive cell death. Mean \pm SD is shown, $n=4$ 
Table 2 Spearman's correlation coefficients between analytes in MLS 402-91 cells applying 70 kinase inhibitors

\begin{tabular}{llllll}
\hline & $\begin{array}{l}\text { Cell } \\
\text { proliferation }\end{array}$ & $\begin{array}{l}\text { FUS-DDIT3 } \\
(\text { mRNA) }\end{array}$ & $\begin{array}{l}\text { FUS } \\
\text { (mRNA) }\end{array}$ & $\begin{array}{l}\text { FUS-DDIT3 } \\
\text { (protein) }\end{array}$ \\
\hline Cell proliferation & 1 & & & \\
FUS-DDIT3 (mRNA) & $0.32^{*}$ & 1 & 1 & 1 \\
FUS (mRNA) & $0.36^{*}$ & $0.93^{*}$ & $0.63^{*}$ & $-0.29 *$ & 1 \\
FUS-DDIT3 (protein) & $0.38^{*}$ & $0.64^{*}$ & $-0.35^{*}$ & \\
FUS (protein) & -0.07 & $-0.35^{*}$ & & \\
\hline
\end{tabular}

$* P<0.0001$, two-tailed significance test

FUS-DDIT3 at the protein level correlated with its mRNA expression, while FUS displayed a negative correlation between its protein and mRNA expression. 18 and 22 inhibitors generated $\geq$ two-fold FUS-DDIT3 and FUS regulation, respectively.

\section{Cell line dependent FUS-DDIT3 and FUS regulation}

To test if the inhibitors displayed similar effect in two additional MLS cell lines, 2645-94 and 1765-92, we selected twelve inhibitors: 2, 6, 7, 9, 10, 11, 13, 18, 19, 20, 27, and 61. The rationale of selecting these inhibitors was that they altered cell proliferation to different degrees and affected FUS-DDIT3 and FUS regulation at the mRNA and protein levels differently. Furthermore, to determine if FUS-DDIT3 and FUS regulation was cell proliferation dependent, we also applied a lower inhibitor concentration $(2.5 \mu \mathrm{M})$.

Figure 4a shows that most inhibitors displayed similar but weaker cell proliferation effects as observed in the initial MLS 402-91 screening experiment. The inhibitor effects were also consistent among the three MLS cell lines. In the initial screen, inhibitor 2, 6, and 7 resulted in $>32$ times reduced cell proliferation, while a lower inhibitor concentration in the follow-up experiment resulted in $<4$ times reduced cell proliferation.

At the mRNA level, the regulation of FUS-DDIT3 and FUS correlated in each cell line (Table 3), but cell line specific regulation was observed for several inhibitors (Fig. 4b). For example, FUS-DDIT3 was downregulated for most inhibitors in MLS 1765-92, while upregulated in MLS 2545-92. FUS-DDIT3 correlated positively to cell proliferation in MLS 1765-92, while FUS correlated positively to cell proliferation in MLS 2645-94 and 1765-92. Supplementary Figure 7 shows that both DNA and RNA were reliable quantified in each condition and MLS cell line using DNA and RNA spike molecules.

Figure $4 \mathrm{c}$ shows that FUS-DDIT3 protein expression was downregulated for most inhibitors and MLS cell lines, while FUS protein expression was divergently regulated. An exception was inhibitor 13 , causing upregulation of both FUS-DDIT3 and FUS in all cell lines. The expression of both FUS-DDIT3 and FUS correlated positively with cell proliferation, as well as to each other (Table 3). Transcript level and protein expression was correlated for FUS-DDIT3 and FUS in all MLS cell lines, except for FUS in MLS 402-91.

Data were overall consistent between the screening and the following-up data sets for MLS 402-91 (Figs. 3 and 4; Tables 2 and 3 ), where the observed regulations were smaller in the follow-up experiments.

\section{Discussion}

Here, we developed a simple approach to quantify DNA, mRNA, and protein in small cell culture samples using direct lysis. A proper direct lysis buffer should disrupt the cell membrane and make analytes accessible. Furthermore, high DNA, RNA, and protein integrity should be maintained whilst the lysis buffer needs to be compatible with downstream enzymatic reactions. Most direct lysis protocols are developed for single-cell applications [2, 15, 16]. In single-cell direct lysis, the major challenge is to minimize molecule losses. In contrast, when the cell number increases, the amount of cell material will eventually inhibit enzymatic reactions. In our approach, mRNA analysis displayed the largest dynamic range followed by DNA and protein analyses. Protein analysis by PLA is known to display a smaller dynamic range than DNA and RNA analyses by qPCR [17]. We speculate that the reduced dynamic range of gDNA compared to mRNA is due to the fact that gDNA becomes less accessible due to less efficient cell lysis when the cell density increases, since gDNA is packed in chromatin which is more challenging to make freely available compared to most mRNAs. A stronger lysis condition, such as the chaotropic agent, guanidinium thiocyanate, could potentially improve lysis of large cell numbers. However, these agents inhibit downstream enzymatic reactions even at weak concentrations $(\sim 100 \mathrm{nM}$ guanidinium thiocyanate inhibits RT) [2, 15]. Hence, samples need to be diluted before being added to enzymatic reactions, reducing its use for small sample sizes.

Typically, mRNA expression is normalized against stably expressed reference genes that are identified by 


\section{A} -Proliferation (gDNA)

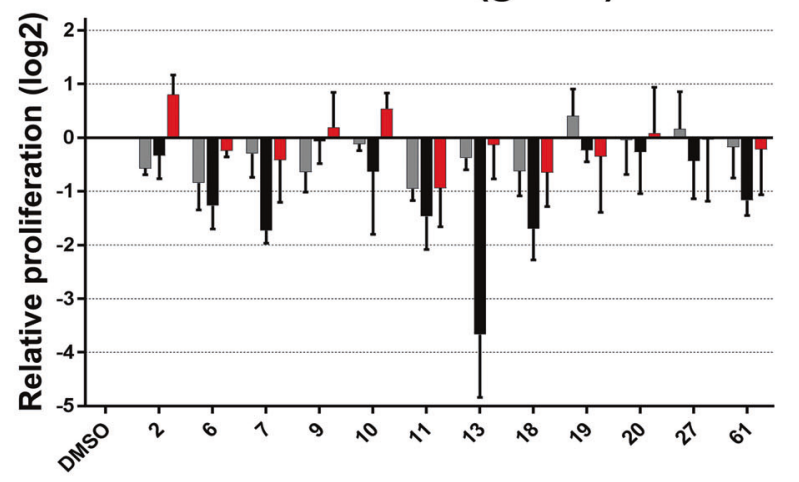

B

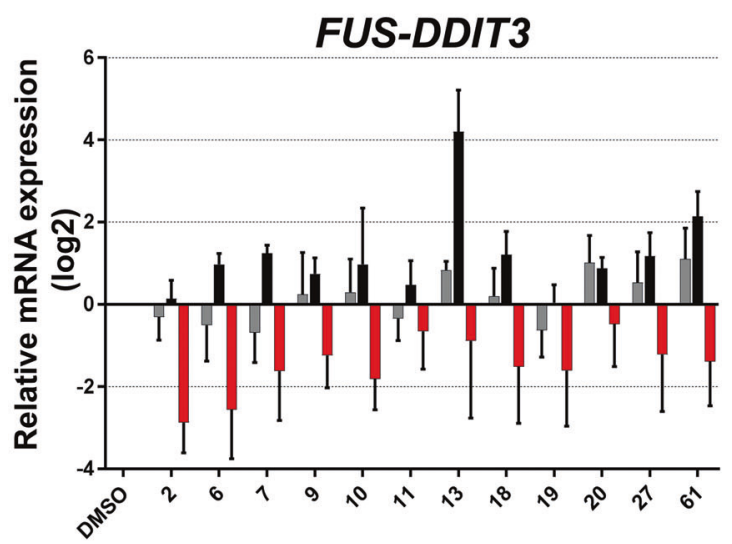

mRNA regulation

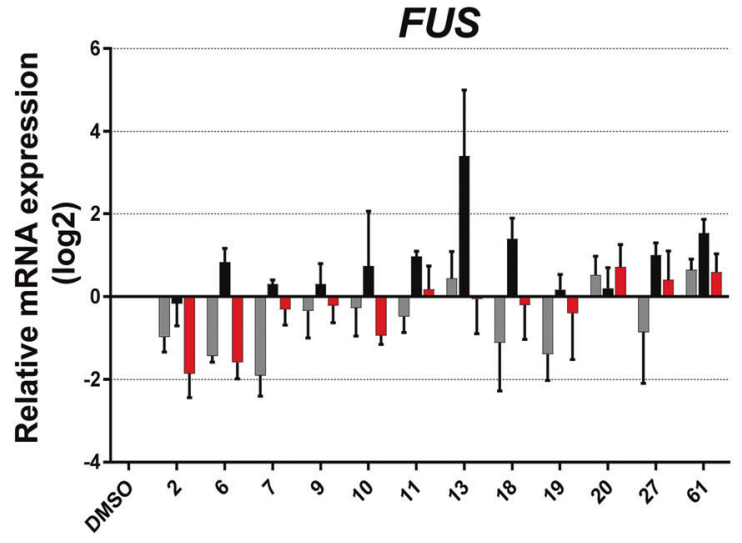

C Protein regulation

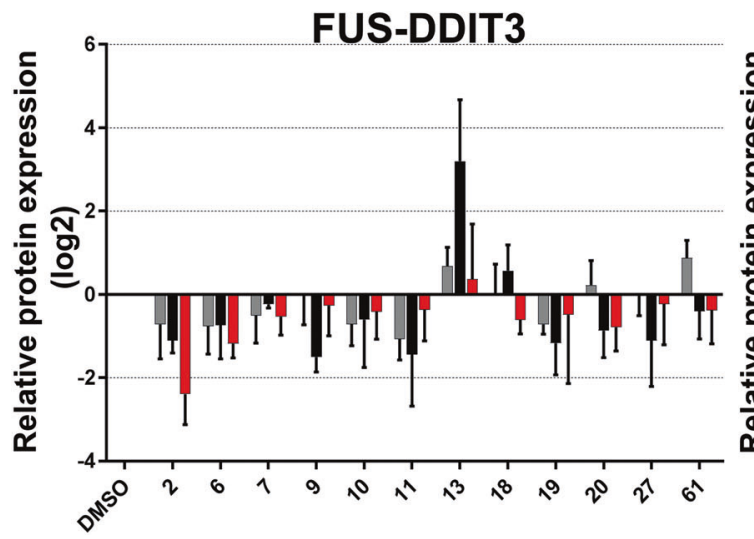

Fig. 4 Kinase inhibitor validation. MLS 402-91, 2645-94 and 1765-92 cells were treated with 12 kinase inhibitors $(2.5 \mu \mathrm{M})$ and compared to treatment controls (DMSO). Cells from three wells on the same 96-well plate were analyzed in three to four independent cell culturing experiments. A Cell proliferation. The DMSO control was arbitrarily set to a value of zero. Different gDNA assays were used for each MLS cell line based on the NormFinder algorithm

statistical algorithms like NormFinder [13]. mRNA is superior to gDNA for normalization of standard samples, since the extraction procedure affects DNA and RNA differently which may introduce quantitative biases [14]. The

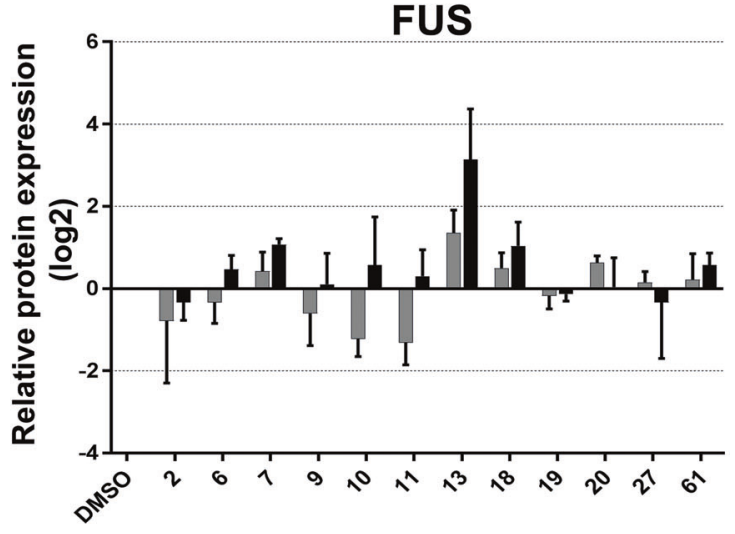

(Supplementary Table 2). Mean \pm SEM is shown, $n=3-4$. B FUS DDIT3 and FUS mRNA expressions. The DMSO controls were arbitrarily set to a value of zero. Mean \pm SEM is shown, $n=3-4$. C FUS-DDIT3 and FUS protein expressions. The DMSO controls were arbitrarily set to a value of zero. Note that FUS alone cannot be quantified with our PLA in MLS 1765-92. Mean \pm SEM is shown, $n=3-4$

strategies to identify constantly expressed reference genes and normalize data globally cannot compensate for variation in the total transcriptome [18]. The total transcriptome size is dependent on several factors, including MYC 
Table 3 Spearman's correlation coefficients between analytes in MLS 402-91, 2645-94, and 1765-92 applying 12 kinase inhibitors

\begin{tabular}{lllll}
\hline & $\begin{array}{l}\text { Cell } \\
\text { proliferation }\end{array}$ & $\begin{array}{l}\text { FUS-DDIT3 } \\
\text { (mRNA) }\end{array}$ & $\begin{array}{l}\text { FUS } \\
(\text { mRNA) }\end{array}$ & $\begin{array}{l}\text { FUS-DDIT3 } \\
\text { (protein) }\end{array}$ \\
\hline Cell proliferation & 1 & & & \\
FUS-DDIT3 (mRNA) & $0.04 / 0.19 / 0.27 *$ & 1 & & \\
FUS (mRNA) & $0.17 / 0.40 * / 0.31 *$ & $0.77 * / 0.63 * / 0.51^{*}$ & 1 & 1 \\
FUS-DDIT3 (protein) & $0.26 * / 0.33 * / 0.42 *$ & $0.33 * / 0.28 * / 0.32 *$ & $0.33 * / 0.43 * / 0.67 *$ & $0.58 * / 0.51 * /$ n.a. \\
FUS (protein) & $0.27 * / 0.47 * /$ n.a. & $0.05 / 0.21 /$ n.a. & $-0.01 / 0.33 * /$ n.a. & 1 \\
\hline
\end{tabular}

Correlation coefficients are listed as MLS 402-91 / MLS 2645-94 / MLS 1765-92

n.a. not applicable

$* P<0.01$, two-tailed test

regulation [19] and cell cycle phase [20, 21]. Direct lysis approaches overcome these obstacles. Furthermore, in our data, gDNA was equally good as reference mRNAs when evaluated by traditional means, i.e., the NormFinder algorithm. Hence, we used gDNA to normalize both mRNA and protein data. A potential limitation of using gDNA as a normalizer is if tumor cells display aneuploidy and instable genomes. Biases caused by these factors should be minimal in MLS, since this tumor entity is genetically stable with an intact TP53 system [22]. In conclusion, direct lysis is simple and cost-effective. Furthermore, alternative data normalization approaches are possible and small sample sizes can be analyzed. The main disadvantage with direct lysis is that the dynamic range to analyze DNA and mRNA is smaller compared to purified samples.

The FET fusion oncogenes, including FUS-DDIT3, are considered important in tumor development and believed to act as abnormal transcription factors [4, 23]. Most studies have focused on FET fusion oncoprotein functions and downstream effects. However, the expression level of oncoproteins is also crucial for many oncogenic functions, with elevated expression causing oncogenic stress, senescence or cell death [24]. Here, we screened 70 kinase inhibitors that target several well-characterized signaling pathways to determine how they affected FUS-DDIT3 regulation at mRNA and protein levels. In addition, we analyzed the expression of the normal FUS allele, since it shares the $5^{\prime}$ promotor region and the $\mathrm{N}$-terminal protein part with FUS-DDIT3. We identified several inhibitors with strong cell proliferation effects in MLS. Overall, we applied a relatively high inhibitor concentration and further doseresponse experiments are needed to determine if some inhibitors alone or in combination could potentially be useful for MLS treatment.

At the mRNA level, FUS-DDIT3 and FUS were strongly co-regulated in all cell lines and experiments. We have previously shown that the FUS-DDIT3 expression level is controlled by the $5^{\prime} F U S$ promotor with the half-life controlled by the DDIT3 sequence [10]. The high correlations between FUS-DDIT3 and FUS indicate that the transcription rate more than the mRNA decay determine the expression levels of the respective gene when regulated under our experimental conditions. Furthermore, all inhibitors caused similar relative regulation of FUS-DDIT3 and FUS regardless of cell proliferation. Hence, this regulation pattern is not a direct effect of cell proliferation rates. Most, but not all, JAK and GSK-3 inhibitors caused FUS-DDIT3 and FUS downregulation, while WNT/ $\beta$-catenin inhibitors generated a small upregulation in the screening experiment (Fig. 3). However, mRNA regulation was cell line dependent (Fig. 4). In the follow-up experiment, GSK-3 inhibitors also showed less downregulation compared to the screening experiment. CHIR99021 (inhibitor 13) even caused an upregulation.

Overall, the FUS-DDIT3 protein regulation correlated with its mRNA level, while the FUS expression level correlated less with its mRNA. We speculate that the lower degree of correlations for FUS is that the FUS half-life is longer than for FUS-DDIT3 [10]. Consequently, downregulation of FUS mRNA will have a delayed response time at protein level compared to FUS-DDIT3. The positive correlation between FUS-DDIT3 and cell proliferation was surprising given that high levels of FUS-DDIT3 causes oncogenic stress and cell cycle arrest. However, most of our inhibitors caused reduced cell proliferation whilst FUS-DDIT3 expression was similar or lower in these conditions compared to control cells. Therefore, we speculate that FUS-DDIT3 expression is favorable for cell proliferation up to a certain level [25, 26], i.e., the FUS-DDIT3 level observed in untreated cells. This hypothesis is supported by a previous report showing that ectopic FUS-DDIT3 expression in MLS cell lines caused reduced cell proliferation [10].

Several inhibitors targeting JAK and GSK-3 caused downregulation of FUS-DDIT3, while no clear trend was observed for FUS. All three MLS cell lines carry different FUS-DDIT3 isoforms and we have previously shown that both protein and transcript half-lives are FUS-DDIT3 isoform dependent. We speculate that the FUS-DDIT3 isoform is one of the reasons behind the observed cell line dependent regulation of FUS-DDIT3 and FUS. To determine the exact effect of specific inhibitors and signaling pathways on 
MLS cell lines and on FUS-DDIT3 and FUS regulation additional mechanistic studies are required.

Acknowledgements This work was supported by grants from Assar Gabrielssons Research Foundation; BioCARE National Strategic Research Program at University of Gothenburg; BIOCEV CZ.1.05/ 1.1.00/02.0109; Johan Jansson Foundation for Cancer Research; Knut and Alice Wallenberg Foundation, Wallenberg Center for molecular and translational medicine, University of Gothenburg, Sweden; Sahlgrenska Academy (ALF) at University of Gothenburg (716321 and 722211); Swedish Cancer Society (2016-438 and 2015-7130); Swedish Research Council (2017-01392); Swedish Society for Medical Research; Swedish Childhood Cancer Foundation (2017-0043); Wilhelm and Martina Lundgren Foundation for Scientific Research and VINNOVA.

\section{Compliance with ethical standards}

Conflict of interest DS and AS declare stock ownership in TATAA Biocenter. MS is an employee of Thermo Fisher Scientific and declares stock ownership in Thermo Fisher Scientific. The remaining authors declare that they have no conflict of interest.

Open Access This article is licensed under a Creative Commons Attribution-NonCommercial-NoDerivatives 4.0 International License, which permits any non-commercial use, sharing, distribution and reproduction in any medium or format, as long as you give appropriate credit to the original author(s) and the source, and provide a link to the Creative Commons license. You do not have permission under this license to share adapted material derived from this article or parts of it. The images or other third party material in this article are included in the article's Creative Commons license, unless indicated otherwise in a credit line to the material. If material is not included in the article's Creative Commons license and your intended use is not permitted by statutory regulation or exceeds the permitted use, you will need to obtain permission directly from the copyright holder. To view a copy of this license, visit http://creativecommons.org/licenses/by-nc-nd/4.0/.

\section{References}

1. Ståhlberg A, Thomsen C, Ruff D, et al. Quantitative PCR analysis of DNA, RNAs, and proteins in the same single cell. Clin Chem. 2012;58:1682-91.

2. Svec D, Andersson D, Pekny M, et al. Direct cell lysis for singlecell gene expression profiling. Front Oncol. 2013;3:274.

3. Åman P. Fusion genes in solid tumors. Semin Cancer Biol. 1999;9:303-18.

4. Riggi N, Cironi L, Suva ML, et al. Sarcomas: genetics, signalling, and cellular origins. Part 1: the fellowship of TET. J Pathol. 2007;213:4-20.

5. Rowley JD, Le Beau MM, Rabbitts TH, editors. Chromosomal translocations and genome rearrangements in cancer. 1st ed. New York, NY: Springer International Publishing Switzerland, 2015, 321-333.

6. Åman P. Fusion oncogenes in tumor development. Semin Cancer Biol. 2005;15:236-43.

7. Engstrom K, Willen H, Kabjorn-Gustafsson C, et al. The myxoid/ round cell liposarcoma fusion oncogene FUS-DDIT3 and the normal DDIT3 induce a liposarcoma phenotype in transfected human fibrosarcoma cells. Am J Pathol. 2006;168:1642-53.
8. Andersson MK, Ståhlberg A, Arvidsson Y, et al. The multifunctional FUS, EWS and TAF15 proto-oncoproteins show cell type-specific expression patterns and involvement in cell spreading and stress response. BMC Cell Biol. 2008;9:37.

9. Jauhiainen A, Thomsen C, Strombom L, et al. Distinct cytoplasmic and nuclear functions of the stress induced protein DDIT3/CHOP/GADD153. PLoS One. 2012;7:e33208.

10. Åman P, Dolatabadi S, Svec D, et al. Regulatory mechanisms, expression levels and proliferation effects of the FUS-DDIT3 fusion oncogene in liposarcoma. J Pathol. 2016;238:689-99.

11. Thelin-Jarnum S, Goransson M, Burguete AS, et al. The myxoid liposarcoma specific TLS-CHOP fusion protein localizes to nuclear structures distinct from PML nuclear bodies. Int J Cancer. 2002;97:446-50.

12. Ståhlberg A, Elbing K, Andrade-Garda JM, et al. Multiway realtime PCR gene expression profiling in yeast Saccharomyces cerevisiae reveals altered transcriptional response of $\mathrm{ADH}$-genes to glucose stimuli. BMC Genom. 2008;9:170.

13. Andersen CL, Jensen JL, Orntoft TF. Normalization of real-time quantitative reverse transcription-PCR data: a model-based variance estimation approach to identify genes suited for normalization, applied to bladder and colon cancer data sets. Cancer Res. 2004;64:5245-50.

14. Bustin SA, Benes V, Garson JA, et al. The MIQE guidelines: minimum information for publication of quantitative real-time PCR experiments. Clin Chem. 2009;55:611-22.

15. Bengtsson M, Hemberg M, Rorsman P, et al. Quantification of mRNA in single cells and modelling of RT-qPCR induced noise. BMC Mol Biol. 2008;9:63.

16. Ståhlberg A, Bengtsson M, Hemberg M, et al. Quantitative transcription factor analysis of undifferentiated single human embryonic stem cells. Clin Chem. 2009;55:2162-70.

17. Fredriksson S, Gullberg M, Jarvius J, et al. Protein detection using proximity-dependent DNA ligation assays. Nat Biotechnol. 2002;20:473-7.

18. Loven J, Orlando DA, Sigova AA, et al. Revisiting global gene expression analysis. Cell. 2012;151:476-82.

19. Lin CY, Loven J, Rahl PB, et al. Transcriptional amplification in tumor cells with elevated c-Myc. Cell. 2012;151:56-67.

20. Dolatabadi S, Candia J, Akrap N, et al. Cell cycle and cell size dependent gene expression reveals distinct subpopulations at single-cell level. Front Genet. 2017;8:1.

21. Karlsson J, Kroneis T, Jonasson E, et al. Transcriptomic characterization of the human cell cycle in individual unsynchronized cells. J Mol Biol. 2017;429:3909-24.

22. Ståhlberg A, Kåbjörn Gustafsson C, Engtröm K, et al. Normal and functional TP53 in genetically stable myxoid/round cell liposarcoma. PLoS One. 2014;9:e113110.

23. Riggi N, Cironi L, Provero P, et al. Expression of the FUS-CHOP fusion protein in primary mesenchymal progenitor cells gives rise to a model of myxoid liposarcoma. Cancer Res. 2006;66:7016-23.

24. Bartkova J, Rezaei N, Liontos M, et al. Oncogene-induced senescence is part of the tumorigenesis barrier imposed by DNA damage checkpoints. Nature. 2006;444:633-7.

25. Oikawa $\mathrm{K}$, Tanaka $\mathrm{M}$, Itoh $\mathrm{S}$, et al. A novel oncogenic pathway by TLS-CHOP involving repression of MDA-7/IL-24 expression. $\mathrm{Br}$ J Cancer. 2012;106:1976-9.

26. Kerr LT, Donoghue JF, Wilding AL, et al. Axitinib has antiangiogenic and antitumorigenic activity in myxoid liposarcoma. Sarcoma. 2016;2016:3484673-17. 